\title{
Mental Toughness in Sport: Motivational Antecedents and Associations with Performance and Psychological Health
}

John W. Mahoney ${ }^{1,3}$, Daniel F. Gucciardi ${ }^{2}$, Nikos Ntoumanis ${ }^{3}$ and Clifford J. Mallett ${ }^{1}$

${ }^{1}$ School of Human Movement Studies, The University of Queensland

${ }^{2}$ School of Physiotherapy and Exercise Science, Curtin University

${ }^{3}$ School of Sport, Exercise, and Rehabilitation Sciences, University of Birmingham

Author Note

Correspondence concerning this article should be addressed to John Mahoney, School of Human Movement Studies, The University of Queensland, St Lucia, Brisbane, QLD, Australia 4067. Telephone: +61 73365 6240. Fax: +61 73365 6877. Email:

j.mahoney@psy.uq.edu.au

To appear in: Journal of Sport \& Exercise Psychology

Accepted for publication: $16^{\text {th }}$ March 2014 
2 We argue that basic psychological needs theory (BPNT) offers impetus to the value of mental

3 toughness as a mechanism for optimizing human functioning. We hypothesized that

4 psychological needs satisfaction (thwarting) would be associated with higher (lower) levels

5 of mental toughness, positive affect, and performance, and lower (higher) levels of negative

6 affect. We also expected that mental toughness would be associated with higher levels of

7 positive affect and performance, and lower levels of negative affect. Further, we predicted

8 that coaching environments would be related to mental toughness indirectly through

9 psychological needs, and that psychological needs would indirectly relate with performance

10 and affect through mental toughness. Adolescent cross-country runners (136 male and 85

11 female, $\left.M_{\mathrm{age}}=14.36\right)$ completed questionnaires pertaining to BPNT variables, mental

12 toughness, and affect. Race times were also collected. Our findings supported our

13 hypotheses. We concluded that BPNT is generative in understanding some of the antecedents

14 and consequences of mental toughness and is a novel framework useful for understanding

15 mental toughness.

16

17 Keywords: Autonomy, Positive Youth Development, Coach Environments, Human

18 Functioning, 
Mental Toughness in Sport: Motivational Antecedents and Associations with Performance and Psychological Health

Mental toughness is a term that is often used to describe a collection of psychological characteristics thought to be central to high performance (Butt, Weinberg, \& Culp, 2010; Jones, Hanton, \& Connaughton, 2002). Over the last decade, researchers have expended considerable efforts in attempting to define and conceptualize mental toughness. As such, there have been recent advancements in understanding this concept. To progress this research field further, there is a need to investigate the positioning of mental toughness within a nomological network of relations that includes variables from established theories within the broad field of psychological enquiry. One such theory proposed in the literature as being connected to mental toughness (Gucciardi \& Mallett, 2010) is self-determination theory (SDT; Deci \& Ryan, 2002). Drawing on theory such as SDT would expand the boundaries of mental toughness research and provide new perspectives in understanding the development and consequences of this concept. The present investigation is a step toward this direction as it aims to examine how mental toughness is linked to motivational variables encompassed by self-determination theory, as well as psychological health, and objective sport performance.

\section{Mental Toughness in Sport}

Gucciardi, Hanton, Gordon, Mallett, and Temby (in press) recently defined mental toughness as a personal capacity to produce consistently high levels of subjective (e.g., personal goal achievement) or objective (e.g., race times) performance despite everyday challenges and stressors as well as significant adversities. This capacity has been discussed as a collection of personal characteristics including attributes such as self-confidence, optimistic thinking, and buoyancy, leading to a general consensus that mental toughness is a multidimensional concept (Butt et al., 2010; Jones et al., 2002). In testing this assumption regarding the dimensionality of mental toughness, Gucciardi et al. (in press) found that there 
was considerable empirical overlap among such personal characteristics and that a

multidimensional construct was limited in terms of discriminant validity. As a result, they proposed and found support for a direct, unidimensional model of mental toughness. They found excellent model fit and good-to-excellent factor loadings for the unidimensional model across three performance groups (i.e., sport, academia, business), as well as strong correlations with theoretically related properties (i.e., perceived stress, performance, goal attainment, thriving). Such evidence highlighted that the personal characteristics reported in previous studies aimed at conceptualizing mental toughness are not readily distinguishable by individuals in performance contexts and therefore called into question the multidimensionality of this concept. Gucciardi et al.'s (in press) work provides a foundation upon which to consider further lines of enquiry that would position mental toughness alongside variables from other theoretical frameworks and help identify associated predictors and outcomes of the concept.

\section{Linking Mental Toughness with SDT}

Although we focus on the links between mental toughness and SDT in this paper, we acknowledge that other theories of motivation (e.g., self-efficacy theory; Bandura, 1977; achievement goal theory; Elliot \& McGregor, 2001) are potentially useful for understanding consistently high performance. For example, in line with self-efficacy theory, the degree to which individuals perceive their actions as efficacious will determine how much effort they expend and for how long they persist on tasks (Bandura, 1977). Similarly, findings from achievement goal theory (e.g., Puente-Diaz, 2012) suggest that effortful and persistent actions are determined by how individuals define (i.e., absolute, intra-individual, or normative) and valance (i.e., positive or negative) notions of competence. These motivational theories evidence strong links with behaviors implicit in Gucciardi et al.'s (in press) definition and,

69 hence, are potentially useful in understanding mental toughness. Despite motivational 
theories such as these holding currency for understanding mental toughness, we focus on SDT in the current study because of previous proposed links between this particular theory and mental toughness (e.g., Gucciardi \& Mallett, 2010), as well as to open debate about the theoretical underpinnings of mental toughness and its development - an avenue researchers have largely neglected in previous research.

Self-determination theory is comprised of five mini-theories, one of which is particularly apt for the present study, namely basic psychological needs theory (BPNT, Deci \& Ryan, 2002). In line with BPNT, the optimization of human functioning is contingent on the degree to which individuals perceive the satisfaction of three fundamental psychological needs: autonomy (the belief that one's actions are self-chosen), competence (the belief that one can bring about desired outcomes), and relatedness (the belief that one is meaningfully connected with a wider social network).

We propose that mental toughness is connected to notions that underscore BPNT as it too concerns the optimization of human functioning in performance contexts. In addition, researchers have shown that BPNT variables are predictive of behaviors or characteristics consistent with the definitional and conceptual properties of mental toughness. For example, there is evidence to support associations between psychological needs satisfaction and persistence (e.g., Pelletier, Fortier, Vallerand, \& Brière, 2001), effort (e.g., Boiché, Sarrazin, Grouzet, Pelletier, \& Chanal, 2008), concentration (e.g., Standage, Duda, \& Ntoumanis, 2003), adaptive coping (e.g., Smith, Ntoumanis, Duda, \& Vansteenkiste, 2011), and challenging-seeking (e.g., Standage et al., 2003).

Other principles detailed in BPNT are also useful for interpreting mental toughness. In particular, within BPNT, psychological needs satisfaction is dependent on the degree to which autonomy, competence, and relatedness are supported by social environments. Social

94 environments that nurture all three psychological needs are termed autonomy-supportive 
(despite the title, autonomy-supportive environments support all three psychological needs), whereas those that thwart psychological needs are termed controlling (Bartholomew, Ntoumanis, \& Thogersen-Ntoumani, 2009; Deci \& Ryan, 2000). Su and Reeves (2011), in their meta-analysis of the extant literature, identified autonomy-supportive environments as being characterized by the offering of choice (within boundaries), the acknowledgement of feelings or perspectives, the use of non-controlling actions and feedback, the provision of meaningful rationales, and the nurturing of individuals' inner motivational resources (e.g., curiosity, enjoyment, belonging). In comparison, controlling environments are characterized by the manipulative use of rewards, negative conditional regard, intimidation, and excessive personal control (Bartholomew, Ntoumanis, \& Thøgersen-Ntoumani, 2010).

In line with previous findings (Bartholomew, Ntoumanis, Ryan, Bosch, \& ThogersenNtoumani, 2011) and recent speculations in the literature (Gucciardi \& Mallett, 2010), we propose that the provision of autonomy-supportive environments may lead to the facilitation of mental toughness, whereas controlling environments may lead to the forestallment of mental toughness. Elucidating these suggestions further, previous findings show that factors believed to be responsible for the development of mental toughness share the characteristics of autonomy-supportive environments. In particular, researchers (e.g., Connaughton, Wadey, Hanton, \& Jones, 2008; Gucciardi, Gordon, Dimmock, \& Mallett, 2009) have suggested that mental toughness development is contingent on athletes being afforded opportunities to explore and engage in tasks volitionally (e.g., self-directed learning), perceiving themselves as competent and feeling challenged during learning (e.g., being able to demonstrate skill mastery, engage in competitive challenges), and feeling respected, cared for, and needed by those around them (e.g., positive social support, a sense of belonging). In line with BPNT, autonomy-supportive environments are key to the optimization of human functioning because 
119 of how they nurture psychological needs satisfaction, suggesting an indirect association

120 between social environments and functioning through psychological needs satisfaction.

As architects of athletes' experiences, coaches are pivotal in the provision of the

social environments that may either foster (i.e., autonomy-supportive) or forestall (i.e.,

123 controlling) mental toughness. Although not explicitly focused on BPNT principles,

124 Gucciardi et al. (2009) proposed that coaches who exhibit behaviors consistent with the notion of autonomy-supportive environments (e.g., encourage athlete input, challenge learning, promote mastery, create non-hostile social environments) were more likely to facilitate mental toughness. Gucciardi et al. (2009) also found that coaches who engage in behaviors consistent with notions of controlling environments (e.g., emphasize ego

129 involvement) are likely to thwart mental toughness development. As articulated above, it is

130 likely that coaching environments are associated with mental toughness indirectly depending

131 on the degree to which such environments nurture individuals' psychological needs.

\section{Linking BPNT to Adaptive Outcomes through Mental Toughness}

Researchers have shown that athletic performance (e.g., Gillet, Vallerand, Amoura, \&

134 Baldes, 2010), as well as positive and negative affect (e.g., Aide, Duda, \& Ntoumanis, 2008) are contingent on the satisfaction of psychological needs that result from the provision of autonomy-supportive environments. Findings from related fields of psychological enquiry

137 provides evidence demonstrating that better athletic performances, higher levels of positive

138 affect, and lower levels of negative affect are associated with the personal characteristics

139 consistent with mental toughness conceptualizations (e.g., self-belief, Caprara, Steca,

140 Gerbino, Paciello, \& Vecchio, 2006; success mindset, Elliot \& McGregor, 2001; emotional

141 awareness and regulation, Salami, 2011). Further, preliminary research has supported

142 theoretically expected relations between mental toughness and performance (Bell, Hardy, \&

143 Beattie, 2013; Gucciardi et al., in press), positive affect, and negative affect (Gucciardi et al., 
144 in press). Given the plausible links and preliminary evidence of relations between mental 145 toughness and BPNT variables, performance, and both positive and negative affect, we

146 contest a nomological network of relations that details the antecedents and outcomes of

147 mental toughness. In particular, we propose that BPNT variables facilitate mental toughness

148 that, in turn, results in adaptive athlete outcomes.

149 The aim of the current study was to explore 1) how motivational variables detailed in 150 BPNT relate to adolescent athletes' mental toughness levels; and 2) the associations between 151 both motivation variables and mental toughness and adaptive outcomes (i.e., performance 152 and positive and negative affect). We were also interested in exploring the indirect relations 153 between coaching environments and mental toughness through psychological needs, as well 154 as the indirect relations between psychological needs and adaptive outcomes through mental toughness. Adolescence was considered because it is a stage of development most commonly associated with interpersonal differences in mental toughness and, therefore, arguably the most pertinent age group to investigate questions of substantive interest (Bell et al., 2013). In line with previous research on BPNT, we predicted that athletes who reported higher levels of autonomy support from their coaches would perceive higher levels of psychological needs satisfaction and lower levels of psychological needs thwarting $(H / a)$. In contrast, higher levels of perceived coach control was expected to be associated with lower levels of psychological needs satisfaction and higher levels of psychological needs thwarting

$163(H 1 b)$. Further, athletes who perceived higher levels of psychological needs satisfaction 164 would report higher levels of positive affect, lower levels of negative affect, and faster race times $(H 2 a)$, whilst greater psychological needs thwarting would be associated with lower levels of positive affect, higher levels of negative affect, and slower race times $(H 2 b)$. Based on the arguments articulated above pertaining to how BPNT variables inform an understanding of mental toughness, we predicted that athletes who perceived higher levels 
169 of psychological needs satisfaction would report higher levels of mental toughness $(H 3 a)$ and

170 athletes who perceived higher levels of psychological needs thwarting would report lower

171 levels of mental toughness $(H 3 b)$. We also predicted that, based on preliminary findings (Bell

172 et al., 2013; Gucciardi et al., in press) athletes who reported higher levels of mental toughness

173 would also report higher levels of positive affect, lower levels of negative affect, and quicker

174 race times compared to adolescent athletes who reported lower levels of mental toughness

175 (H4). These hypothesized direct relations can are illustrated in Figure 1. Finally we made several predictions pertaining to indirect relations. We predicted that autonomy-supportive coaching environments would be positively $(\mathrm{H} 5 \mathrm{a})$ and controlling environments would be

178 negatively $(H 5 b)$ related with mental toughness through psychological needs satisfaction.

179 Conversely, we expected that autonomy-supportive coaching environments would be negatively $(H 5 c)$ and controlling environments would be positively $(H 5 d)$ related with mental toughness through psychological needs thwarting. We also expected that psychological needs satisfaction would be positively (H6a) and psychological needs thwarting would be negatively $(H 6 b)$ associated with positive affect through mental toughness, whilst psychological needs satisfaction would be negatively (H6c) and psychological needs thwarting would be positively $(H 6 d)$ associated with negative affect and race times through mental toughness.

\section{Method}

\section{Participants}

Participants were 136 male $\left(M_{\text {age }}=14.39, S D=1.44\right)$ and 85 female $\left(M_{\text {age }}=14.29\right.$,

$190 S D=1.53)$ cross-country runners recruited from high schools in Australia $(N=221)$. On

191 average, participants had been competing in inter-school cross-country events for 4.47 years

$192(S D=2.57)$ and trained 2.10 hours per week $(S D=1.63)$.

\section{Measures}


Demographics. Participants' age, gender, years competing in cross-country, and number of training hours per week were garnered using single item measures.

Mental Toughness Index (MTI). The MTI (Gucciardi et al., in press) is an eight-

197 item direct measure of mental toughness (e.g., "I am able to regulate my focus when

198 performing tasks"). Each question represents one of the eight facets of mental toughness

199 proposed in Gucciardi et al.'s (2011) synthesis of the literature. Participants respond to each item on a 7 -point scale $(1=$ false, $100 \%$ of the time and 7 true, $100 \%$ of the time $)$. The scale has received psychometric support with samples of university students, athletes, and employees, and theoretically consistent relations with performance, stress, and psychological health (Gucciardi et al., in press).

Sport Climate Questionnaire - Short Form (SCQ-SF). The SCQ-SF is a sportadaption of the Learning Climate Questionnaire (Williams \& Deci, 1996), which measures athletes' perceptions of coach autonomy support (e.g., "I feel that my coach provides me with choices and options"). Participants respond to the 6-item questionnaire using a scale ranging from 1 (strongly disagree) to 7 (strongly agree). The SCQ-SF has been validated in sport samples (e.g., Hagger, Chatzisarantis, Culverhouse, \& Biddle, 2003).

Basic Needs Satisfaction in Sport Scale (BNSSS). The BNSSS (Ng, Lonsdale, \&

211 Hodge, 2011) measures athletes' perceptions of competence (e.g., "I am skilled at my sport"),

212 relatedness (e.g., "I show concern for others in my sport”), and autonomy. Ng et al.'s (2011)

213 measure separates autonomy into three categories, namely volition (e.g., "I feel I participate

214 in my sport willingly"), choice (e.g., "In my sport, I get opportunities to make choices"), and

215 internal perceived locus of causality (e.g., "In my sport, I feel I am pursuing goals that are my

216 own"). Participants respond on a scale ranging from 1 (not at all true) to 7 (very true).

217 Psychometric analyses showed the 20-item measure to have satisfactory internal consistency 
scores and model fit, and good nomological validity and test-retest reliability ( $\mathrm{Ng}$ et al., 2011).

Psychological Needs Thwarting Scale (PNTS). The PNTS (Bartholomew,

221 Ntoumanis, Ryan, \& Thogersen-Ntoumani, 2011) is a 12-item measure of athletes’

222 perceptions of psychological needs thwarting. This measure includes statements pertaining to

223 the thwarting of autonomy (e.g., "I feel pushed to behave in certain ways"), competence (e.g.,

224 "There are situations where I am made to feel inadequate"), and relatedness (e.g., "I feel rejected by those around me"), and requires participants to respond on a seven-point scale (1

$226=$ strongly disagree and $7=$ strongly agree . Analyses have revealed support for the three-

227 factor model and internal consistency (Bartholomew et al., 2011).

Controlling Coach Behaviors Scale (CCBS). The CCBS (Bartholomew et al., 2010) includes 15 items pertaining to athletes' perceptions of their coaches' behaviors. The scale is comprised of four factors: controlling use of rewards (e.g., "my coach only rewards/praises me to make me train harder"), negative conditional regard (e.g., "my coach pays me less attention if I have displeased him/her"), intimidation (e.g., "my coach threatens to punish me to keep me in line during training"), and excessive personal control (e.g., "my coach tries to control what I do during my free time"). Reponses are rated on a 7-point scale from 1 (strongly disagree) to 7 (strongly agree). Statistical analyses have revealed sound content and factorial validity for the measure, as well as internal consistency and invariance across gender and sport type (Bartholomew et al., 2010).

Psychological health. Positive affect was measured using the Mental Health

239 Continuum Short Form (MHC-SF, Keyes, 2005). This 14-item questionnaire requires

240 individuals to indicate the degree to which they have experienced certain thoughts and

241 feelings over the past month on a 6-point scale $(1=$ never and $6=$ every day $)$. Questions are 242 categorized into three factors, emotional (e.g., "happy"), psychological (e.g., "that your life 
243 has a sense of direction or meaning to it"), and social (e.g., "that people are basically good").

244 High internal consistency scores and evidence of discriminatory validity support the use of 245 the MHC-SF (Keyes, 2005).

The 21-item Depression Anxiety Stress Scale (DASS-21) was employed to measure

247 negative affect (Lovibond \& Lovibond, 1995). The DASS-21 measures depression (e.g., "I

248 felt down-hearted and blue"), anxiety (e.g., "I felt I was close to panic"), and stress (e.g., "I

249 found it difficult to relax"), and requires individuals to respond on a 4-point scale $(0=$ did not apply to me, $1=$ applied to me to some degree, or some of the time, $2=$ applied to me a considerable degree, or a good part of time, and $3=$ applied to me very much, or most of the time). The DASS-21 has been shown to have strong factor loadings, discriminator validity, and internal consistency (Antony, Bieling, Cox, Enns, \& Swinson, 1998).

Performance. Race times over varying distances (depending on age and gender) were collected during the end-of-season championship and served as a measure of performance.

This event was selected because of the high attendance of athletes and because mental toughness is thought to be most pertinent during pressure-filled performances such as end-ofseason championships (Bell et al., 2013). Race times were standardized to account for differences in race distance across age and gender (e.g., 15 year old boys ran 4 km, 15 year old girls ran $3 \mathrm{~km}$ ). A higher race time equated to poorer performance.

\section{Procedure}

Following university ethics approval, school staff (i.e., principals and/or sport directors) were approached and informed about the aims and procedures of the research. Information sheets and written consent forms were then distributed to parents/guardians and adolescent athletes during training sessions. Once parent/guardian and participant written consent was received, participants were asked to complete a booklet that included the

267 abovementioned questionnaires. Participants completed the questionnaires roughly one 
month before the end-of-season inter-school championship. The demographic questions appeared first in all booklets and the remaining questionnaires were randomly counterbalanced. Race times were recorded during the championship event by race organizers.

\section{Data Analysis}

Path analysis with a Bayesian estimator was applied in Mplus 7.11 (Muthén \&

273 Muthén, 1998-2012) to examine the hypothesized model depicted in Figure 1 (for general examples and descriptions of Bayesian analysis see, van de Schoot et al., in press; Zyphur \& Oswald, in press) including both direct and indirect pathways (see, Yuan \& MacKinnon, 2009). Bayesian analysis is an approach that has garnered the interests of sport and exercise psychology researchers in recent years (Doran \& Gaudreau, 2014; Jackson, Gucciardi, \& Dimmock, 2014). This approach leverages off theory and previous research to form a prior distribution - a combination of the specific magnitude and variability of effect sizes. Prior distributions are then incorporated into the analysis to determine the probability of a hypothesized model, given the data (Muthén \& Asparouhov, 2012). Prior distributions can range from non-informative, where no prior knowledge is asserted about the magnitude or variance of the parameter, to highly informative, where the distribution is constrained by very precise parameter estimates. These prior distributions are combined with new data to form the posterior distribution - an updated understanding of the prior distribution in light of the given data. In totality, all available evidence - prior and current - is considered in the process of Bayesian analysis. Additionally, Bayesian analysis does not depend on asymptotic (largesample) theory and, as such, provides more accurate estimates of parameters and model fit than frequentist approaches when sample size is small. Another benefit of Bayesian analysis over traditional approaches is that it is more flexible when handling complex models, as the use of prior knowledge incorporates additional information into the analysis that help identify 
292 parameter solutions that otherwise might not be achieved by using a frequentist approach

293 (Asparouhov \& Muthén, 2012, July 18).

294 We used both empirical evidence and theoretical knowledge to guide the specification

295 of priors in our analysis. First, prior knowledge regarding the relations between coaching

296 climate and psychological needs, and psychological needs and psychological health were

297 guided by empirical evidence (Bartholomew et al., 2011). We utilized Bartholomew et al.'s

298 findings because of the similarity between the aims, sample, and measures of their study and

299 ours. For similar reasons, we utilized Gucciardi et al.'s (in press) findings to inform the

300 selection of priors for the relations between mental toughness and both positive and negative

301 affect. The empirically informed priors and their respective variances can be seen in Table 1.

302 Although the effects of both BPNT variables (Gillet et al., 2010) and mental

303 toughness (Bell et al., 2013; Gucciardi et al., in press) on performance have been examined in

304 previous research, it is difficult and often inappropriate to guide priors when exploring

305 unrelated performances (e.g., mean performances in closed sports are not equivalent to mean

306 performances in endurance sports). Hence, drawing on statistical recommendations (Muthén

307 \& Asparouhov, 2012; Zyphur \& Oswald, in press) and theoretical expectations, the priors for

308 the effects of psychological needs satisfaction/thwarting on mental toughness were set with a

309 mean of -.40 and a variance of .03 , meaning that $95 \%$ of the loadings should fall between -.06

310 and -.74 . These means and variances were selected to reflect the expected direction of

311 relations between mental toughness and race times (i.e., inverse relations), as informed by

312 past research, whilst limiting constraints on the strength between these associations (for

313 further details about the use and selection of theoretically informed priors see, Zyphur \&

314 Oswald, in press). As the use of different priors can influence the relations between variables

315 (Zyphur \& Oswald, in press), we conducted a sensitivity analysis by comparing the

316 hypothesized model (i.e., informed by empirical and theoretical priors) with two other 
models; one with the same mean parameters but with variances around the expected parameter estimates set to be highly precise, and another with low precision for the variance of the parameter distribution (see Table 1). with Bayesian modeling. Bayesian analysis employs a sophisticated estimation process known as Markov Chain Monte Carlo (MCMC) whereby the prior distribution is specified and through an iterative process an accurate representation of the posterior distribution is approximated from representative samples of parameter values from the entire posterior distribution (for detailed discussions about MCMC methods and application, see Chen, Shao, \& Ibrahim, 2000; Gamerman \& Lopes, 2006). At least two MCMC estimation "chains" are run in parallel, each using different starting values for model parameters to ensure the iterative process provides an opportunity to monitor convergence (Muthén \& Asparouhov, 2012). Two diagnostic tools can be created from these chains: (i) the potential scale reduction (PSR) factor, which takes into account the overall parameter variability both within and between the chains; and (ii) trace plots, which graphically represent the fluctuation in parameter values as the MCMC estimator iterates toward the solution. A PSR value of $\leq 1.1$ provides evidence in support of convergence to the true posterior distribution, as it suggests that parameter variability could not be appreciably reduced with further iterations (Asparouhov \& Muthén, 2010, September 29). Visual inspection of trace plots should indicate that the multiple independent chains have all stabilized to essentially the same distribution (Asparouhov \& Muthén, 2010, September 29).

Model fit is subsequently assessed using posterior predictive checking (for more detail, see Lynch \& Western, 2004). This method compares the probability of the observed

340 data against that of the generated posterior distribution of parameters, while taking into 341 account variability in the parameters. Specifically, the posterior predictive $p$ (PPP) value 
342 indicates the degree of deviation between the observed and generated data and is

343 accompanied by a 95\% confidence interval. In line with recommendations (Muthén \&

344 Asparouhov, 2012), PPP values closer to .50 reflect good fitting models where the real data is

345 just as probable as the generated data and, as such, should be preferred when comparing

346 competing models.

347 Throughout our analyses we considered parameters to have gained substantive

348 support when the $95 \%$ credibility interval (95\% CI) did not encompass zero. It is necessary to

349 note that credibility intervals are different from the more common confidence intervals from

350 Frequentist approaches. Both credibility and confidence intervals service a similar aim: to

351 provide the best estimate of the true nature of the parameter. However, credibility intervals

352 incorporate prior knowledge into the estimate and represent an estimation of the probability

353 that the true value of a parameter falls between two bounds (i.e., upper and lower intervals),

354 whereas confidence intervals are based solely on the data and estimate a range in which the

355 parameter would occur over time with repeated sampling (Curran, 2005). In interpreting

356 credibility intervals, researchers can conclude, for example, that they are $95 \%$ certain that the

357 true value of the parameter exists between the upper and lower bounds. In comparison,

358 researchers interpreting confidence intervals could conclude that, on average, $95 \%$ of

359 intervals generated via repeated sampling would contain the true value of the parameter (for

360 further discussions, see, Curran, 2005)

\section{Results}

Table 2 includes descriptive statistics, reliability scores, and correlations of the study variables and relevant demographic markers. Model convergence was supported through a smooth decrease in PSR values at the first iteration and PSR stability once $<1.1$ was reached, as well as visual inspection of trace plot (these results are extensive and are not included in

366 this manuscript, but are available from the first author upon request). All three models (see 
367 Table 1) demonstrated sound fit indices. In light of these results, and in keeping with prior 368 findings, we focus our discussions on the hypothesized model (i.e., Model A). Bayesian estimates and $95 \%$ CIs for the associations between the study variables for

370 all three models are summarized in Table 1. Theoretically consistent relations were evidenced

371 between social environments and psychological needs. In particular, autonomy-supportive

372 environments were positively associated with psychological needs satisfaction and negatively

373 associated with psychological needs thwarting. Further, controlling environments were

374 positively associated with psychological needs thwarting and negatively related with

375 psychological needs satisfaction. Psychological needs were also strongly associated with

376 mental toughness, as well as positive and negative affect, and performance. Specifically,

377 psychological needs satisfaction was positively associated with mental toughness and

378 positive affect, and negatively associated with negative affect and race times. Further, psychological needs thwarting was positively associated with negative affect and race times, and negatively associated with mental toughness and positive affect. Finally, mental toughness was strongly associated with positive and negative affect, and race times as hypothesized. Specifically, mental toughness was positively related to positive affect and negatively associated with negative affect and race times. environments and mental toughness, as well as the relations between controlling environments and mental toughness. Similarly, psychological needs thwarting mediated the

387 relations between autonomy-supportive environments and mental toughness, as well as controlling environments and mental toughness. Further, mental toughness mediated the relations between psychological needs satisfaction and positive and negative affect, and

390 performance, as well as psychological needs thwarting and positive and negative affect, and 391 performance (Table 3). 
is a concept that can be positioned within a nomological network of relations that provides an insight into to its motivation antecedents and relations with performance and psychological outcomes. The aims of the current study were to explore 1) how motivational variables detailed in BPNT relate to adolescent athletes' mental toughness levels and 2) the associations between both motivation variables and mental toughness and adaptive outcomes (i.e., performance and positive and negative affect). We were also interested in exploring how coaching environments and mental toughness were indirectly related through psychological needs, as well as how psychological needs and adaptive outcomes were indirectly associated through mental toughness.

In the first instance, all direct relations between the coaching climate and psychological needs $(H 1 a-b)$, and between psychological needs and outcome variables $(H 2 a-$ $b$ ) were supported. These findings compliment previous research that has identified associations between social environments and psychological needs, and between psychological needs and outcome variables (Deci \& Ryan, 2000; Ntoumanis, 2012). Beyond these results, the major substantive findings of our study pertain to the direct and indirect associations involving mental toughness, which highlight a nomological network within which this concept can be understood. To our knowledge, this study is the first to show that

411 psychological needs satisfaction is positively, whilst psychological needs thwarting is

412 inversely associated with mental toughness $(H 3 a-b)$. Arguably, to produce consistently

413 higher levels of performance despite obstacles faced - that is, to demonstrate greater levels of

414 mental toughness - individuals need to not only expend a great deal of cognitive and

415 behavioral effort, but also maintain this effort over time. In line with BPNT, the quality and 416 quantity of cognitive and behavior effort available to individuals is contingent on the degree 
417 to which psychological needs are satisfied (Deci \& Ryan, 2000). That is, psychological needs

418 satisfaction promotes perceptions of personal control, self-efficacy, and self-value that result

419 in the maintenance of high levels of effort. In comparison, psychological needs thwarting

420 inhibits individuals' sense of personal control, efficaciousness, and importance, resulting in a

421 reduction or forfeiting of effort - behaviors that reflect lower levels of mental toughness.

422 We also found that mental toughness levels were positively associated with positive

423 affect and inversely associated with negative affect and race times $(H 4)$. These relations are

424 consistent with preliminary evidence in sport (Bell et al., 2013; Gucciardi et al., in press).

425 Further, these data provide additional support for Gucciardi et al.'s (in press) definition of

426 mental toughness (i.e., that higher levels of mental toughness are representative of better

427 performances) and helps shore up the conceptual foundations of this concept by highlighting

428 meaningful associations. However, there are numerous avenues that researchers need to

429 consider before firmer conclusions can be drawn about the adaptive potential of mental

430 toughness. A recommendation previously presented in the literature (Andersen, 2011)

431 concerns the perceptions and actions of injured athletes who are more mentally tough. It is

432 possible that such individuals would jeopodize their recovery by ignoring feelings of pain and

433 not adhere to rehabilitation recommedations in order to pursue competition goals, meaning

434 that mental toughness is maladaptive in particular contexts. Researchers could investigate

435 such contexts to further explore whether or not mental toughness is solely adaptive or also

436 relates to maladaptive outcomes.

437 We also found support for the expected indirect association between coaching

438 environments and mental toughness through psychological needs (H5a-d). These findings are

439 consistent with a body of previous research which has shown environmental supports and

440 outcome variables to be indirectly related through psychological needs (e.g., Bartholomew,

441 Ntoumanis, Ryan, Bosch, et al., 2011). However, our findings are unique as they are, to our 
442 knowledge, the first to identify associations between BPNT variables and mental toughness.

443 Our findings extend on previous research by Gucciardi et al. (2009) who reported that

444 different coaching styles can foster or forestall mental toughness development. We agree with

445 Gucciardi et al.'s (2009) conclusions, but also extend them by contesting that the degree to

446 which coaching environments nurture psychological needs is one mechanism through which

447 coaches may contribute to mental toughness development.

448 A final substantive finding of our study was the indirect relations between

449 psychological needs and adaptive outcomes through mental toughness $(H 6 a-d)$. Above we

450 proposed that psychological needs satisfaction promoted continuous, high effort because of

451 an increased sense of personal control, efficaciousness, and self-value, and that this was

452 reflective of mental toughness. We extended this line of thinking by suggesting that higher

453 levels of continuous effort are more likely to result in individuals feeling as though they are

454 mastering new skill, goal achievement, and a sense of productivity and, as such is likely to

455 enhance perceptions of positive affect. The opposite could be said of individuals who expend

456 little effort on tasks because their psychological needs are thwarted. That is, less effort is

457 likely to result in stagnation, underachievement, and reduced productivity and, as such, is

458 likely to produce greater levels of negative affect.

459 Some shortcomings of the current study offer possible avenues for future research.

460 The first notable limitation was the use of a cross-sectional methodology. The use of

461 longitudinal methods in subsequent studies would allow researchers to monitor changes in

462 social environments, psychological needs, mental toughness, and markers of human

463 functioning (e.g., positive affect, performance). Another possible methodological avenue to

464 overcome the cross-sectional limitation of the current study would be to conduct an

465 experimental trial where coaches are exposed to a training program aimed at fostering more

466 autonomy-supportive and less controlling interpersonal styles. Athletes' perceptions of 
coaching behaviors, psychological needs satisfaction, and mental toughness could then be monitored at the end of the intervention and at follow-ups to determine the causal effects of BPNT variables on mental toughness. A second limitation of the current study was the sole emphasis on coaching environments. Coaching environments were selected in the current study because of their prevalence in previous mental toughness literature (e.g., Connaughton et al., 2008; Gucciardi et al., 2009), but also because coaches often form strong relationships with adolescents as they emancipate from their primary caregivers (Jowett \& TimsonKatchis, 2005). Nevertheless, parents and peers are two other groups identified as playing a meaningful role in the provision of autonomy-supportive or controlling environments $(\mathrm{Su} \&$ Reeve, 2011), as well as mental toughness development (e.g., Connaughton et al., 2008). Researchers could explore how other social agents contribute to psychological needs, mental toughness, and associated outcomes. A third limitation of this study concerns the manner in which prior distributions in the Bayesian analysis were informed. Specifically, a single source informed the selection of some priors, whereas others were theoretically informed. We acknowledge that ideally these priors would have been informed by point and variance estimates of effect sizes obtained from meta-analyses and that it is impossible to account for variability across contexts with such sparse prior knowledge. In line with changing trends in statistical enquiry and the growing interests in Bayesian approaches in particular, we suggest that researchers continue to add to the pool of available data on topics such as mental toughness in order to allow substantiated conclusions to be formed. Finally, as alluded to in the introduction of this paper, SDT is but one lens through which to consider mental toughness and its development. Other theories such as self-efficacy theory (Bandura, 1977) and achievement goal theory (Elliot \& McGregor, 2001) may be useful for understanding mental toughness and its development and should be considered in subsequent research. 
492 understanding mental toughness. They provide new insight into how motivational variables

493 proposed by BPNT are linked to mental toughness and highlight a conceptual model that

494 helps researchers to understand some of the antecedents and consequence of mental

495 toughness. Conceptually, we believe findings such as those reported in this study advances

496 mental toughness research by directing it into a new wave of enquiry. Further exploration

497 along these lines is required to offer a more comprehensive understanding of the positioning

498 of mental toughness amongst other psychological concepts and its value in supporting

499 optimal human functioning. 


\section{References}

Aide, J. W., Duda, J. L., \& Ntoumanis, N. (2008). Autonomy support, basic need satisfaction and optimal functioning of adult male and female sport participants: A test of basic needs theory. Motivation and Emotion, 32, 189-199. doi: 10.1007/s11031-008-9095-z

Andersen, M. B. (2011). Who's mental, who's tough and who's both? Mutton constructs dressed up as lamb. In D. F. Gucciardi \& S. Gordon (Eds.), Mental toughness in sport: Developments in theory and research (pp. 69-88). Abingdon, UK: Routledge.

Antony, M. M., Bieling, P. J., Cox, B. J., Enns, M. W., \& Swinson, R. P. (1998).

Psychometric properties of the 42-item and 21-item versions of the Depression Anxiety Stress Scales in clinical groups and a community sample. Psychological Assessment, 10, 176-181. doi: 10.1037//1040-3590.10.2.176

Asparouhov, T., \& Muthén, B. (2010, September 29). Bayesian analysis using Mplus: Technical implementation. Retrieved February 27, 2014, from http://www.statmodel.com/download/Bayes3.pdf

Asparouhov, T., \& Muthén, B. (2012, July 18). General random effect latent variable modeling: Random subjects, items, contexts, and parameters. Retrieved February 27, 2014, from http://www.statmodel.com/download/NCME12.pdf

Bandura, A. (1977). Self-efficacy: Toward a unifying theory of behavioral change. Psychological Review, 84, 191-215. doi: 10.1037//0033-295X.84.2.191

Bartholomew, K. J., Ntoumanis, N., Ryan, R. M., Bosch, J. A., \& Thogersen-Ntoumani, C. (2011). Self-determination theory and diminished functioning: The role of interpersonal control and psychological need thwarting. Personality and Social Psychology Bulletin, 37, 1449-1473. doi: 10.1177/0146167211413125 
Bartholomew, K. J., Ntoumanis, N., Ryan, R. M., \& Thogersen-Ntoumani, C. (2011). Psychological need thwarting in the sport context: Assessing the dark side of athletic experience. Journal of Sport \& Exercise Psychology, 33, 75-102.

Bartholomew, K. J., Ntoumanis, N., \& Thogersen-Ntoumani, C. (2009). A review of controlling motivational strategies from a self-determination theory perspective: Implications for sports coaches. International Review of Sport and Exercise Psychology, 2, 215-233. doi: 10.1080/17509840903235330

Bartholomew, K. J., Ntoumanis, N., \& Thøgersen-Ntoumani, C. (2010). The controlling interpersonal style in a coaching context: Development and initial validation of a psychometric scale. Journal of Sport \& Exercise Psychology, 32, 193-216.

Bell, J. J., Hardy, L., \& Beattie, S. (2013). Enhancing mental toughness and performance under pressure in elite young cricketers: A 2-year longitudinal intervention. [Advance online publication]. Sport, Exercise, and Performance Psychology. doi: $10.1037 / \mathrm{a} 0033129$

Boiché, J. C. S., Sarrazin, P. G., Grouzet, F. M. E., Pelletier, L., \& Chanal, J. P. (2008). Students' motivational profiles and achievement outcomes in physical education: A self-determination perspective. Journal of Educational Psychology, 100, 688-701. doi: $10.1037 / 0022-0663.100 .3 .688$

Butt, J., Weinberg, R., \& Culp, B. (2010). Exploring mental toughness in NCAA athletes. Journal of Intercollegiate Sport, 3, 316-332.

Caprara, G. V., Steca, P., Gerbino, M., Paciello, M., \& Vecchio, G. M. (2006). Looking for adolescents' well-being: Self-efficacy beliefs as determinants of positive thinking and happiness. Epidemiologia e Psichiatria Sociale, 15, 30-43. doi: $10.1017 / \mathrm{S} 1121189 \mathrm{X} 00002013$ 
Chen, M. H., Shao, Q. M., \& Ibrahim, J. G. (2000). Monte Carlo methods in Bayesian computation. New York: Springer-Verlag.

Connaughton, D., Wadey, R., Hanton, S., \& Jones, G. (2008). The development and maintenance of mental toughness: Perceptions of elite performers. Journal of Sport Sciences, 26, 83-95. doi: 10.1080/02640410701310958

Curran, J. M. (2005). An introduction to Bayesian credibility intervals for sampling error in DNA profiles. Law, Probability and Risk, 4, 115-126. doi: doi:10.1093/lpr/mgi009

Deci, E. L., \& Ryan, R. M. (2000). The "what" and "why" of goal pursuits: Human needs and the self-determination of behavior. Psychological Inquiry, 11, 227-268. doi: 10.1207/S15327965PLI1104_01

Deci, E. L., \& Ryan, R. M. (2002). Self-determination research: Reflections and future directions. In E. L. Deci \& R. M. Ryan (Eds.), Handbook of self-determination research (pp. 431-441). Rochester, NY: University of Rochester Press.

Doran, J., \& Gaudreau, P. (2014). A point-by-point analysis of performance in a fencing match: Psychological processes associated with winning and losing streaks. Journal of Sport \& Exercise Psychology, 36, 3-13. doi: 10.1123/jsep.2013-0043

Elliot, A. J., \& McGregor, H. A. (2001). A 2 x 2 goal achievement framework. Journal of Personality and Social Psychology, 80, 501-519. doi: 10.1037//0022-3514.80.3.501

Gamerman, D., \& Lopes, H. F. (2006). Markov Chain Monte Carlo: Stochastic simulation for Bayesian inference (2nd ed.). Boca Raton, FL: Chapman \& Hall/CRC.

Gillet, N., Vallerand, R. J., Amoura, S., \& Baldes, B. (2010). Influence of coaches' autonomy support on athletes' motivation and sport performance: A test of the hierarchical model of intrinsic and extrinsic motivation. Psychology of Sport and Exercise, 11, 155-161. doi: 10.1016/j.psychsport.2009.10.004 
Gucciardi, D. F., Gordon, S., Dimmock, J., \& Mallett, C. J. (2009). Understanding the coach's role in the development of mental toughness: Perspectives of elite Australian football coaches. Journal of Sport Sciences, 27, 1483-1496. doi:

\section{$10.1080 / 02640410903150475$}

Gucciardi, D. F., Hanton, S., Gordon, S., Mallett, C. J., \& Temby, P. (in press). The concept and measurement of mental toughness: Test of dimensionality, nomological network and traitness. Journal of Personality.

Gucciardi, D. F., \& Mallett, C. J. (2010). Understanding and developing mental toughness in sport. In S. J. Hanrahan \& M. B. Andersen (Eds.), Handbook of applied sport psychology (pp. 547-556). London: Routledge.

Hagger, M. S., Chatzisarantis, N., Culverhouse, T., \& Biddle, S. J. H. (2003). The processes by which perceived autonomy support in physical education promotes leisure-time physical activity intentions and behaviour: A trans-contextual model. Journal of Educational Psychology, 95, 784-795. doi: 10.1037/0022-0663.95.4.784

Jackson, B., Gucciardi, D. F., \& Dimmock, J. (2014). Toward a multidimensional model of athletes' commitment to coach-athlete relationships and interdependent sport teams: A substantive-methodological synergy. Journal of Sport \& Exercise Psychology, 36, $52-$ 68. doi: 10.1123/jsep.2013-0038.

Jones, G., Hanton, S., \& Connaughton, D. (2002). What is this thing called mental toughness? An investigation of elite sport performers. Journal of Applied Sport Psychology, 14, 205-218. doi: 10.1080/10413200290103509

Jowett, S., \& Timson-Katchis, M. (2005). Social networks in sport: Parental influence on the coach-athlete relationship. The Sport Psychologist, 19, 267-287.

Keyes, C. L. (2005). The subjective well-being of America's youth: Toward a comprehensive assessment. Adolescent and Family Health, 4, 3-11. 
Lovibond, S. H., \& Lovibond, P. F. (1995). Manual for the Depression Anxiety Stress Scale (2nd ed.). Sydney: Psychology Foundation.

Lynch, S. M., \& Western, B. (2004). Bayesian posterior predictive checks for complex models. Sociological Methods \& Research, 32, 301-335. doi:

$10.1177 / 0049124103257303$

Muthén, B. O., \& Asparouhov, T. (2012). Bayesian structural equation modeling: A more flexible representation of substantive theory. Psychological Methods, 17, 313-335. doi: $10.1037 / \mathrm{a} 0026802$

Muthén, L. K., \& Muthén, B. O. (1998-2012). Mplus user's guide (7th ed.). Los Angeles, CA: Muthén \& Muthén.

Ng, J. Y. Y., Lonsdale, C., \& Hodge, K. (2011). The Basic Needs Satisfaction in Sport Scale (BNSSS): Instrument development and initial validity evidence. Psychology of Sport and Exercise, 12, 257-264. doi: 10.1016/j.psychsport.2010.10.006

Ntoumanis, N. (2012). A self-determination theory perspective on motivation in sport and physical education: Current trends and possible future research directions. In G. C. Roberts \& D. C. Treasure (Eds.), Motivation in sport and exercise (Vol. 3, pp. 91128). Champaign, IL: Human Kinetics.

Pelletier, L. G., Fortier, M. S., Vallerand, R. J., \& Brière, N. M. (2001). Associations among perceived autonomy support forms of self-regulation and persistence: A prospective study. Motivation and Emotion, 25, 279-306. doi: 10.1023/A:1014805132406

Puente-Diaz, R. (2012). The effect of achievement goals on enjoyment, effort, satisfaction and performance. International Journal of Psychology, 47, 102-110. doi: $10.1080 / 00207594.2011 .585159$ 
Salami, S. O. (2011). Personality and psychological well-being of adolescents: The moderating role of emotional intelligence. Social Behavior and Personality, 39, 785794. doi: 10.2224/sbp.2011.39.6.785

Smith, A., Ntoumanis, N., Duda, J. L., \& Vansteenkiste, M. (2011). Goal striving, coping, and well-being in sport: A prospective investigation of the self-concordance model. Journal of Sport \& Exercise Psychology, 33, 124-145.

Standage, M., Duda, J. L., \& Ntoumanis, N. (2003). A model of contextual motivation in physical education: Using constructs from self-determination and achievement goal theories to predict physical activity intentions. Journal of Educational Psychology, 95, 97-110. doi: 10.1037/0022-0663.95.1.9

Su, Y. L., \& Reeve, J. (2011). A meta-analysis of the effectiveness of intervention programs designed to support autonomy. Educational Psychology Review, 23, 159-188. doi: $10.1007 / \mathrm{s} 10648-010-9142-7$

van de Schoot, R., Denissen, J., Neyer, F. J., Kaplan, D., Asendorpf, J. B., \& van Aken, M. A. G. (in press). A gentle introduction to Bayesian analysis: Application to development research. Child Development. doi: 10.1111/cdev.12169

Williams, G. C., \& Deci, E. L. (1996). Internalization of biopsychosocial values by medical students: A test of self-determination theory. Journal of Personality and Social Psychology, 70, 767-779. doi: 10.1037//0022-3514.70.4.767

Yuan, Y., \& MacKinnon, D. P. (2009). Bayesian mediation analysis. Psychological Methods, 14, 301-322. doi: 10.1037/a0016972

Zyphur, M. J., \& Oswald, F. L. (in press). Bayesian estimation and inference: A user's guide. Journal of Management. doi: 10.1177/0149206313501200 
Table 1

Comparison of Unstandardised Weights of Parameter Estimates of Bayesian Estimates using

Different Priors, including Prior Means and Variances of Hypothesized Model

\begin{tabular}{|c|c|c|c|c|}
\hline & Hypothesized Model & Model A & Model B & Model C \\
\hline \multicolumn{5}{|l|}{ Model Fit } \\
\hline PPP (95\% CI) & & $.43[-25.00,29.47]$ & $.43[-25.06,29.37]$ & $.43[-25.19,29.84]$ \\
\hline Parameters & Prior Mean (Variance) & $\mu[95 \% \mathrm{PPI}]$ & $\mu[95 \% \mathrm{PPI}]$ & $\mu[95 \% \mathrm{PPI}]$ \\
\hline $\mathrm{AS} \rightarrow \mathrm{NS}$ & $.46(.03)$ & $.45[.18, .74]^{*}$ & $.46[.40, .52]^{*}$ & $.43[-.07, .95]$ \\
\hline $\mathrm{AS} \rightarrow \mathrm{NT}$ & $-.22(.01)$ & $-.24[-.42,-.05]^{*}$ & $-.22[-.28,-.16]^{*}$ & $-.32[-.81, .17]$ \\
\hline $\mathrm{CO} \rightarrow \mathrm{NS}$ & $-.07(.001)$ & $-.07[-.13,-.01]^{*}$ & $-.07[-.13,-.01]^{*}$ & $-.08[-.78, .69]$ \\
\hline $\mathrm{CO} \rightarrow \mathrm{NT}$ & $.50(.03)$ & $.50[.18, .81]^{*}$ & $.50[.44, .56]^{*}$ & $.50[-.24,1.25]$ \\
\hline $\mathrm{NS} \rightarrow \mathrm{MT}$ & $.40(.03)$ & $.43[.14, .72]^{*}$ & $.40[.34, .46]^{*}$ & $.47[-.02, .96]$ \\
\hline $\mathrm{NS} \rightarrow \mathrm{PA}$ & $.66(.03)$ & $.48[.18, .79]^{*}$ & $.65[.59, .71]^{*}$ & $.30[-.34, .95]$ \\
\hline $\mathrm{NS} \rightarrow \mathrm{NA}$ & $-.16(.005)$ & $-.15[-.28,-.02]^{*}$ & $-.15[-.21,-.09]^{*}$ & $-.12[-.78, .44]$ \\
\hline $\mathrm{NS} \rightarrow \mathrm{RT}$ & $-.40(.03)$ & $-.39[-.72,-.05]^{*}$ & $-.40[-.46,-.34]^{*}$ & $-.35[-1.20, .46]$ \\
\hline $\mathrm{NT} \rightarrow \mathrm{MT}$ & $-.40(.03)$ & $-.37[-.70,-.05]^{*}$ & $-.40[-.46,-.34]^{*}$ & $-.31[-.99, .39]$ \\
\hline $\mathrm{NT} \rightarrow \mathrm{PA}$ & $-.10(.001)$ & $-.10[-.17,-.04]^{*}$ & $-.10[-.17,-.04]^{*}$ & $-.18[-.88, .52]$ \\
\hline $\mathrm{NT} \rightarrow \mathrm{NA}$ & $.24(.01)$ & $.22[.04, .41]^{*}$ & $.24[.18, .30]^{*}$ & $.14[-.43, .85]$ \\
\hline $\mathrm{NT} \rightarrow \mathrm{RT}$ & $.40(.03)$ & $.38[.05, .72]^{*}$ & $.40[.34, .46]^{*}$ & $-.29[-.51,1.13]$ \\
\hline $\mathrm{MT} \rightarrow \mathrm{PA}$ & $.57(.03)$ & $.39[.09, .69]^{*}$ & $.56[.50, .62]^{*}$ & $.21[-.45, .90]$ \\
\hline $\mathrm{MT} \rightarrow \mathrm{NA}$ & $-.18(.005)$ & $-.18[-.31,-.05]^{*}$ & $-.18[-.24,-.12]^{*}$ & $-.20[-.81, .40]$ \\
\hline $\mathrm{MT} \rightarrow \mathrm{RT}$ & $-.40(.03)$ & $-.39[-.72,-.05]^{*}$ & $-.40[-.46,-.34]^{*}$ & $-.35[-1.22, .48]$ \\
\hline
\end{tabular}

Note. Model A = originally hypothesized model; Model B = variance around the expected parameter estimates of original model was set to be highly precise (i.e., .001 or a $95 \%$ limit of \pm .06 around the mean); Model $\mathrm{C}=$ variance around the expected parameter estimates of original model was specific with low precision (i.e., .20 or a $95 \%$ limit of \pm .87 around the mean). $\mathrm{AS}=$ autonomy support; $\mathrm{CO}=$ controlling; $\mathrm{NS}=$ needs satisfaction; NT $=$ needs thwarting; $\mathrm{MT}=$ mental toughness; $\mathrm{PA}=$ positive affect $\mathrm{NA}=$ negative affect $; \mathrm{RT}=$ race times.

*CI did not encompass zero 
Table 2

Descriptive Statistics, Reliability Scores, and Correlations for all Study Variables

\begin{tabular}{|c|c|c|c|c|c|c|c|c|c|c|c|c|c|c|}
\hline & Variables & $M(S D)$ & Skew. & Kurt. & 1 & 2 & 3 & 4 & 5 & 6 & 7 & 8 & 9 & 10 \\
\hline 1 & Age & $14.36(1.47)$ & & & - & & & & & & & & & \\
\hline 2 & Years & $4.47(2.57)$ & & & $0.22 * *$ & - & & & & & & & & \\
\hline 3 & Hrs/wk & $2.10(1.63)$ & & & 0.02 & 0.08 & - & & & & & & & \\
\hline 4 & AS & $5.27(1.16)$ & -.74 & .61 & $0.27 * *$ & 0.12 & $0.16^{*}$ & $(.88)$ & & & & & & \\
\hline 5 & $\mathrm{CO}$ & $2.22(0.92)$ & .77 & .31 & $-0.15^{*}$ & 0.01 & 0.05 & $-0.32 * *$ & $(.88)$ & & & & & \\
\hline 6 & NS & $5.53(0.80)$ & -.60 & .39 & 0.07 & 0.03 & $0.20 * *$ & $0.53 * *$ & $-0.26^{* *}$ & $(.89)$ & & & & \\
\hline 7 & NT & $2.57(1.05)$ & .53 & -.21 & $-0.23 * *$ & -0.07 & -0.05 & $-0.52 * *$ & $0.58 * *$ & $-0.40 * *$ & $(.88)$ & & & \\
\hline 8 & MT & $5.48(0.78)$ & -.63 & 1.00 & 0.06 & -0.01 & $0.18 * *$ & $0.31 * *$ & $-0.24 * *$ & $0.59 * *$ & $-0.38 * *$ & $(.79)$ & & \\
\hline 9 & PA & $4.97(0.74)$ & -1.34 & 2.59 & 0.05 & 0.05 & 0.05 & $0.33 * *$ & -0.13 & $0.46^{* *}$ & $-0.34 * *$ & $0.40 * *$ & $(.90)$ & \\
\hline 10 & NA & $0.53(0.41)$ & 1.18 & 1.36 & $-0.30 * *$ & -0.07 & -0.06 & $-0.23^{* *}$ & $0.25^{* *}$ & $-0.29 * *$ & $0.43 * *$ & $-0.37 * *$ & $-0.38 * *$ & $(.84)$ \\
\hline 11 & Race time & $0.00(0.98)^{\dagger}$ & .71 & .26 & -0.02 & $-0.21 * *$ & $-0.22 * *$ & $-0.16^{*}$ & -0.04 & $-0.22 * *$ & $0.43^{* *}$ & $-0.21 * *$ & 0.02 & 0.08 \\
\hline
\end{tabular}

Note. Skew = Skewtosis; Kurt = Kurtosis Years = years competing in cross-country; Hrs/wk = hours per week spent training in cross-country; AS = autonomy-supportive environments; $\mathrm{CO}=$ controlling coaching environments; NS = psychological needs satisfaction; $\mathrm{NT}=$ psychological needs thwarting; $\mathrm{MT}=$ mental toughness; $\mathrm{PA}=$ positive affect; NA = negative affect; Race time = performance times standardized across age, gender, and distance run; internal reliability estimates (Cronbach's alpha) provided on the diagonal in parentheses.

$* p<.05 . * * p<.01 .{ }^{\dagger}$ Z-scores, race time standardized across age, gender, and distance run. 


\section{Table 3}

\section{Unstandardized Weights of Parameter Estimates for Indirect Effects of Variables in Model A}

Mediation variable

Estimate $(S E)$ 95\% PPI

Indirect path

Needs satisfaction

Autonomy-supportive $\rightarrow$ Mental toughness

Controlling $\rightarrow$ Mental toughness

Needs thwarting

Autonomy-supportive $\rightarrow$ Mental toughness

$0.08(.02)$

$-0.17(.02)$

Controlling $\rightarrow$ Mental toughness

Mental toughness
Need satisfaction $\rightarrow$ Race time
$-0.16(.04)$
$[-0.39,-0.01]^{*}$
Need thwarting $\rightarrow$ Race time
$0.13(.02)$
$[0.01,0.37]^{*}$
Need satisfaction $\rightarrow$ Negative affect
$-0.07(.02)$
$[-0.17,-0.01]^{*}$
Need thwarting $\rightarrow$ Negative affect
$0.06(.01)$
$[0.01,0.16]^{*}$
Needs satisfaction $\rightarrow$ Positive affect
$0.16(.03)$
$[0.02,0.38]^{*}$
Needs thwarting $\rightarrow$ Positive affect
$-0.13(.01)$
$[-0.34,-0.01]^{*}$

Note. $\mathrm{SE}=$ standard error, $\mathrm{PPI}=$ posterior probability interval.

$* \mathrm{CI}$ did not encompass zero. 


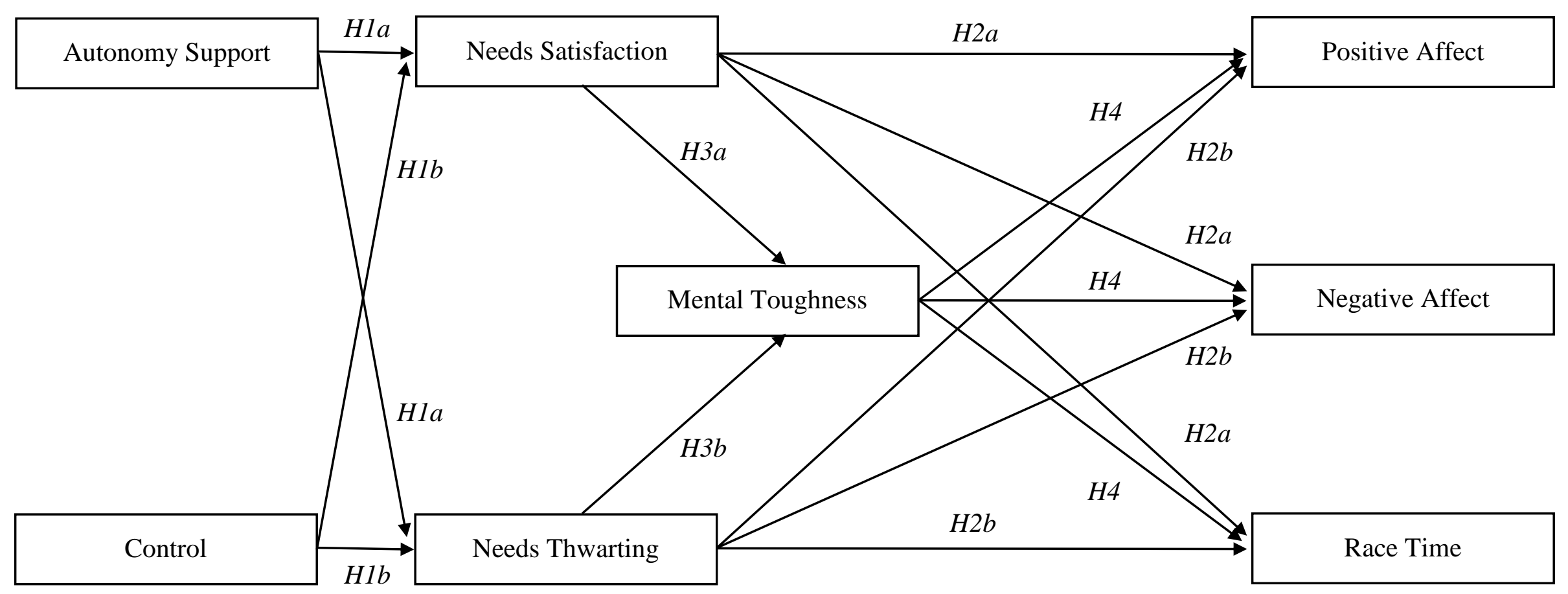

Figure 1. Hypothesized direct relations between coaching environments, psychological needs, mental toughness, performance, positive affect, and negative affect. 